Supporting information

\title{
Practical Synthesis of Optically Active $\alpha, \alpha$-Disubstituted Malonamic Acids through Asymmetric Hydrolysis of Malonamide Derivatives with Rhodococcus sp. CGMCC 0497
}

\author{
Zhong-Liu $\mathrm{Wu}$ and $\mathrm{Zu}-\mathrm{Yi} \mathrm{Li} *$ \\ State Key Laboratory of Bio-organic \& Natural Products Chemistry \\ Shanghai Institute of Organic Chemistry, Chinese Academy of Sciences \\ 354 Fenglin Road, Shanghai 200032 (PRC)
}

\section{Materials and Methods}

The commercially available reagents were used without further purification. Polarimetry was carried out using an optical activity Perkin Elmer 241ML polarimeter and the measurements were made at the sodium D-line with a $10 \mathrm{~cm}$ pathlength cell. Concentrations(c) are given in g/100ml. Enantiomeric excesses: Chiral HPLC was conducted with a PE NELSON NCI900 using Chiralpak AS or Chiralcel OJ column at a flow rate of $0.7 \mathrm{ml} / \mathrm{min}$ with 2-propanol/hexane as the mobile phase.

\section{$\underline{\text { Microorganism and Cultivation }}$}

The strain Rodococcus sp. CGMCC 0497 is available in CGMCC (China General Microbiological Culture Colletion Center). Rodococcus sp. CGMCC 0497 was subcultured at $30^{\circ} \mathrm{C}$ for 24 hours in a $100 \mathrm{ml}$ shaking flask containing $20 \mathrm{ml}$ of a medium consisting of $0.5 \mathrm{~g}$ of polypepton, $0.5 \mathrm{~g}$ of beef extract and $1 \mathrm{~g}$ of glucose per $100 \mathrm{ml}$ of tap water, $\mathrm{pH} 7.0$. Then the subculture was inoculated into a 51 shaking flask containing 11 of the rich medium consisting of $1 \mathrm{~g}$ of glucose, $0.5 \mathrm{~g}$ of beef extract, $0.25 \mathrm{~g}$ of methacrylamide, $100 \mathrm{mg}$ of $\mathrm{K}_{2} \mathrm{HPO}_{4} .3 \mathrm{H}_{2} \mathrm{O}, 75 \mathrm{mg}$ of $\mathrm{KH}_{2} \mathrm{PO}_{4}, 10 \mathrm{mg}$ of $\mathrm{NaCl}, 0.1 \mathrm{ml}$ of mineral medium per $100 \mathrm{ml}$ of tap water with methacrylamide added 24 hours later. The $\mathrm{pH}$ of each medium was adjusted to around 7.0-7.2 by addition of $2 \mathrm{~N} \mathrm{NaOH}$ or $3 \mathrm{~N} \mathrm{HCl}$. After incubation at $30^{\circ} \mathrm{C}$ with reciprocal shaking for $48 \mathrm{hr}$. The organism was harvested by centrifugation using an HIMAC centrifuge CR20B2 (Hitachi, Japan) with a RPR9-2 rotor $\left(6800 \mathrm{~g}, 30 \mathrm{~min} 10^{\circ} \mathrm{C}\right)$. Cells were washed with $100 \mathrm{mM}$ potassium phosphate buffer $(\mathrm{pH}=7.0)$ and centrifugated.

2-benzyl-2-methyl-malonitrile 1. To a suspension of $\mathrm{NaH}(60 \%) 400 \mathrm{mg}(10 \mathrm{mmol})$ in $40 \mathrm{ml}$ THF and 4ml DMF, was added drop by drop a solution of malonitrile $(1.32 \mathrm{~g}$, $20 \mathrm{mmol}$ ) in $5 \mathrm{ml}$ THF at room temperature. After $2 \mathrm{~h}$, benzyl bromide or chloride (10mmol) in THF (10ml) was added within $2 \mathrm{~h}$. Stirring was continued at room temperature for $10 \mathrm{~h}$. The reaction was quenched by aqueous $\mathrm{NH}_{4} \mathrm{Cl}$ solution, extracted with ethyl acetate, dried on $\mathrm{MgSO}_{4}$ and purified by flash chromatograph (74\%). The product was dissolved in acetone $(50 \mathrm{ml})$. Methyl iodide $(20 \mathrm{mmol})$ and solid $\mathrm{K}_{2} \mathrm{CO}_{3}(25 \mathrm{mmol})$ were added. The mixture was stirred overnight at room temperature. After filtration and concentration, the residue was purified by flash chromatograph to yield $\alpha, \alpha$-disubstituted malonitriles $(98 \%)$. white solid, m.p. $94.3-95.3^{\circ} \mathrm{C}$, Lit. ${ }^{1}$ 94.5-95.5 ${ }^{\circ} \mathrm{C}$, ${ }^{1} \mathrm{H}$ NMR $\left(300 \mathrm{MHz}, \mathrm{CDCl}_{3}\right): \delta 7.35-$ 
7.45(m, 5H, Ar-H), 3.22(s, 2H, $\left.\mathrm{CH}_{2}\right), 1.81\left(\mathrm{~s}, 3 \mathrm{H}, \mathrm{CH}_{3}\right)$; IR (KBr): 2251(CN), 765, 703; MS $\mathrm{m} / \mathrm{z}(\%): 170\left(\mathrm{M}^{+}, 1\right), 91(100)$.

\section{Diamide Synthesis}

To a suspension of $\mathrm{NaH}(60 \%) 400 \mathrm{mg}(10 \mathrm{mmol})$ in $40 \mathrm{ml} \mathrm{THF}$ and $4 \mathrm{ml} \mathrm{DMF}$, was added drop by drop a solution of malonic acid diester $(1.32 \mathrm{~g}, 20 \mathrm{mmol})$ in $5 \mathrm{ml}$ THF at room temperature. After $2 \mathrm{~h}$, benzyl bromide or chloride $(10 \mathrm{mmol})$ in THF $(10 \mathrm{ml})$ was added within $2 \mathrm{~h}$. Stirring was continued at room temperature or at gentle reflux for 3-10 h. The reaction was quenched by aqueous $\mathrm{NH}_{4} \mathrm{Cl}$ solution, extracted with ethyl acetate, dried on $\mathrm{MgSO}_{4}$ and purified by flash chromatograph. The product was dissolved in acetone $(50 \mathrm{ml})$. Methyl iodide (20mmol) and solid $\mathrm{K}_{2} \mathrm{CO}_{3}$ or $\mathrm{KOH}(25 \mathrm{mmol})$ were added. The mixture was stirred overnight at room temperature. After filtration and concentration, the residue was purified by flash chromatograph to yield $\alpha, \alpha$-disubstituted malonic acid diesters.

To a solution of $\alpha, \alpha$-disubstituted malonic acid diester $(4.5 \mathrm{mmol})$ in ethanol $(15 \mathrm{ml})$ was added $2 \mathrm{~N} \mathrm{KOH} 15 \mathrm{ml}$ at room temperature. The reaction was continued at $50^{\circ} \mathrm{C}$ for $10-30$ hours and quenched by $3 \mathrm{~N} \mathrm{HCl}$, extracted with ethyl acetate, dried on $\mathrm{MgSO}_{4}$ and purified by flash chromatograph. The product was dissolved in $\mathrm{SOCl}_{2}(3 \mathrm{ml})$ and heated to gentle reflux. After 6 hours, the mixture was added slowly to ice-cold stronger ammonia water and the stirring was continued overnight. After filtration and recrystallization, give a white solid of $\alpha, \alpha$-disubstituted malonamide.

2-benzyl-2-methyl-malonamide 4a. white solid, m.p. $195.4-196.4^{\circ} \mathrm{C}$, Lit. $^{2} 202-203^{\circ} \mathrm{C}$; ${ }^{1} \mathrm{H}$ NMR (300 MHz, DMSO): $\delta$ 7.22-7.12(m, 9H, ArH, 2NH$), 3.06\left(\mathrm{~s}, 2 \mathrm{H}, \mathrm{CH}_{2}\right), 1.10(\mathrm{~s}$, $\left.3 \mathrm{H}, \mathrm{CH}_{3}\right)$; IR(KBr): v 3389, 3205(NH), 1692, 1663(C=O); $\mathrm{MS} \mathrm{m/z}(\%): 206\left(\mathrm{M}^{+}, 7\right)$, 189(10), 162( $\left.\mathrm{M}^{+}-\mathrm{CONH}_{2}, 100\right), 160(41)$.

2-(2'-chrolobenzyl)-2-methyl-malonamide 4b. white solid, m.p. $227.0-228.0{ }^{\circ} \mathrm{C} ;{ }^{1} \mathrm{H}$ NMR (300 MHz, DMSO): $\delta$ 7.41-7.20(m, 8H, Ar-H, 2NH $), 3.27\left(\mathrm{~s}, 2 \mathrm{H}, \mathrm{CH}_{2}\right), 1.11(\mathrm{~s}, 3 \mathrm{H}$, $\left.\mathrm{CH}_{3}\right)$; IR(KBr): $v$ 3433, 3381, 3216(NH), 1647(C=O); $\mathrm{MS} \mathrm{m} / z(\%): 205\left(\mathrm{M}^{+}-\mathrm{Cl}, 100\right)$, 198(13), 196( $\mathrm{M}^{+}-\mathrm{CONH}_{2}$, 37); Anal. Calcd. for $\mathrm{C}_{11} \mathrm{H}_{13} \mathrm{~N}_{2} \mathrm{O}_{2} \mathrm{Cl}: \mathrm{C}, 54.89 ; \mathrm{H}, 5.44 ; \mathrm{N}$, 11.64; Cl, 14.73. Found: C, 54.90; H, 5.43; N, 11.66; Cl, 14.54 .

2-(3'-chrolobenzyl)-2-methyl-malonamide 4c. white solid, m.p. $195.4-196.4^{\circ} \mathrm{C} ;{ }^{1} \mathrm{H}$ NMR (300 MHz, DMSO): $\delta$ 7.26-7.13(m, 8H, ArH, 2NH $), 3.06\left(\mathrm{~s}, 2 \mathrm{H}, \mathrm{CH}_{2}\right), 1.11(\mathrm{~s}, 3 \mathrm{H}$, $\left.\mathrm{CH}_{3}\right) ; \mathrm{IR}(\mathrm{KBr}): v$ 3379, 3213(NH), 1689, $1671(\mathrm{C}=\mathrm{O}) ; \mathrm{MS} \mathrm{m} / \mathrm{z}(\%): 242\left(\mathrm{M}^{+}+2,4\right)$, 240( $\left.\mathrm{M}^{+}, 11\right), 198(33), 196\left(\mathrm{M}^{+}-\mathrm{CONH}_{2}, 100\right)$; Anal. Calcd. for $\mathrm{C}_{11} \mathrm{H}_{13} \mathrm{~N}_{2} \mathrm{O}_{2} \mathrm{Cl}: \mathrm{C}, 54.89 ; \mathrm{H}$, 5.44; N, 11.64; Cl, 14.73. Found: C, 54.62; H, 5.17; N, 11.51; Cl, 14.64.

2-(4'-chrolobenzyl)-2-methyl-malonamide 4d. white solid, m.p. $244.6-245.6{ }^{\circ} \mathrm{C} ;{ }^{1} \mathrm{H}$ NMR (300 MHz, DMSO): $\delta$ 7.29(d, 2H, J=8.7Hz, Ar-H), 7.19-7.16(m, 6H, ArH, 2NH $)$, 3.04(s, 2H, $\left.\mathrm{CH}_{2}\right), 1.10\left(\mathrm{~s}, 3 \mathrm{H}, \mathrm{CH}_{3}\right)$; IR( $\left.\mathrm{KBr}\right): v$ 3387, 3202(NH), 1648(C=O); $\mathrm{MS} \mathrm{m} / \mathrm{z}(\%)$ : 242( $\left.\mathrm{M}^{+}+2,8\right), 240\left(\mathrm{M}^{+}, 23\right), 198(31), 196(100)$; Anal. Calcd. for $\mathrm{C}_{11} \mathrm{H}_{13} \mathrm{~N}_{2} \mathrm{O}_{2} \mathrm{Cl}: \mathrm{C}$, 54.89; $\mathrm{H}, 5.44 ; \mathrm{N}, 11.64 ; \mathrm{Cl}, 14.73$. Found: C, 54.76; H, 5.23; N, 11.55; Cl, 14.70 .

2-(4'-methylbenzyl)-2-methyl-malonamide 4e. white solid, m.p. $246.9-247.9^{\circ} \mathrm{C} ;{ }^{1} \mathrm{H}$ NMR (300 MHz, DMSO): $\delta$ 7.19(s, 2H, NH 2$), 7.12\left(\mathrm{~s}, 2 \mathrm{H}, \mathrm{NH}_{2}\right), 7.03(\mathrm{~s}, 4 \mathrm{H}, \mathrm{ArH}), 3.00(\mathrm{~s}$, 
$\left.2 \mathrm{H}, \mathrm{CH}_{2}\right), 1.08\left(\mathrm{~s}, 3 \mathrm{H}, \mathrm{CH}_{3}\right) ; \mathrm{IR}(\mathrm{KBr}):$ v 3387, 3206(NH), 1689, 1647(C=O); $\mathrm{MS} \mathrm{m} / \mathrm{z}(\%)$ : 220( $\left(\mathrm{M}^{+}, 39\right)$, 176(100), 159(49), 105(94); Anal. Calcd. for $\mathrm{C}_{12} \mathrm{H}_{16} \mathrm{~N}_{2} \mathrm{O}_{2}$ : C, 65.43; H, 7.32; $\mathrm{N}, 12.72$. Found: C, 65.32; H, 7.50; N, 12.72 .

2-(4'-methoxybenzyl)-2-methyl-malonamide 4f. white solid, m.p. $239.3-240.3{ }^{\circ} \mathrm{C} ;{ }^{1} \mathrm{H}$ NMR $(300 \mathrm{MHz}, \mathrm{DMSO}): \delta$ 7.17(s, 2H, $\left.\mathrm{NH}_{2}\right), 7.10\left(\mathrm{~s}, 2 \mathrm{H}, \mathrm{NH}_{2}\right), 7.05,6.77(\mathrm{AB}, 4 \mathrm{H}$, $\mathrm{J}=8.7 \mathrm{~Hz}, \mathrm{ArH}), 3.86(\mathrm{~s}, 3 \mathrm{H}, \mathrm{OMe}), 2.97\left(\mathrm{~s}, 2 \mathrm{H}, \mathrm{CH}_{2}\right), 1.07\left(\mathrm{~s}, 3 \mathrm{H}, \mathrm{CH}_{3}\right) ; \mathrm{IR}(\mathrm{KBr}): \vee 3358$, 3203(NH), 1649(C=O); MS m/z (\%): 236( $\left.\mathrm{M}^{+}, 16\right), 192(17), 121(100)$; HRMS: Calcd. for $\left(\mathrm{C}_{12} \mathrm{H}_{16} \mathrm{~N}_{2} \mathrm{O}_{3}\right)^{+} 236.11609$, Found: 236.11829 .

2-(4'-florobenzyl)-2-methyl-malonamide 4g. white solid, m.p. $206.1-207.1^{\circ} \mathrm{C} ;{ }^{1} \mathrm{H}$ NMR (300 MHz, DMSO): $\delta$ 7.21-7.03(m, 8H, ArH, 2NH $), 3.04\left(\mathrm{~s}, 2 \mathrm{H}, \mathrm{CH}_{2}\right), 1.09(\mathrm{~s}, 3 \mathrm{H}$, $\left.\mathrm{CH}_{3}\right) ; \mathrm{IR}(\mathrm{KBr}): v$ 3392, 3203(NH), 1694, 1649(C=O); MS m/z (\%): 224(M $\left.{ }^{+}, 10\right), 207(3)$, 180(49), 109(100); Anal. Calcd. for $\mathrm{C}_{11} \mathrm{H}_{13} \mathrm{~N}_{2} \mathrm{O}_{2} \mathrm{~F}$ : C, 58.92; H, 5.84; N, 12.49; F, 8.47. Found: C, 58.75; H, 5.72; N, 12.54; F, 8.05.

2-(4'-bromobenzyl)-2-methyl-malonamide 4h. white solid, m.p. $251.5-252.5^{\circ} \mathrm{C} ;{ }^{1} \mathrm{H}$ NMR (300 MHz, DMSO): $\delta$ 7.42, 7.11(AB, 4H, J=8.4Hz, ArH), 7.21(s, 2H, $\left.\mathrm{NH}_{2}\right), 7.16(\mathrm{~s}$, $\left.2 \mathrm{H}, \mathrm{NH}_{2}\right), 3.03\left(\mathrm{~s}, 2 \mathrm{H}, \mathrm{CH}_{2}\right), 1.10\left(\mathrm{~s}, 3 \mathrm{H}, \mathrm{CH}_{3}\right) ; \mathrm{IR}(\mathrm{KBr}): \vee 3391,3201(\mathrm{NH}), 1689(\mathrm{C}=\mathrm{O})$, 1647; MS $m / z(\%): 286\left(\mathrm{M}^{+}+2,11\right), 284\left(\mathrm{M}^{+}, 11\right), 242(80), 240(100)$; Anal. Calcd. for $\mathrm{C}_{11} \mathrm{H}_{13} \mathrm{~N}_{2} \mathrm{O}_{2} \mathrm{Br}: \mathrm{C}$, 46.34; H, 4.60; N, 9.82; Br, 28.02. Found: C, 46.40; H, 4.62; N, 9.77; $\mathrm{Br}, 27.98$.

2-phenylethyl-2-methyl-malonamide 4i. white solid, m.p. $169.3-170.3^{\circ} \mathrm{C} ;{ }^{1} \mathrm{H}$ NMR (300 MHz, DMSO): $\delta$ 7.30-7.11(m, 9H, $\left.\mathrm{ArH}, 2 \mathrm{NH}_{2}\right), 2.47-2.41\left(\mathrm{~m}, 2 \mathrm{H}, \mathrm{CH}_{2}\right), 2.02-1.96(\mathrm{~m}$, 2H, CH2), 1.32(s, 3H, $\left.\mathrm{CH}_{3}\right)$; IR(KBr): v 3417, 3371, 3250, 3214(NH), 1652(C=O); MS $m / z$ (\%): 220(M $\left.\mathrm{M}^{+}, 0.1\right), 219(1), 116(100)$, 99(30), 91(33); Anal. Calcd. for $\mathrm{C}_{12} \mathrm{H}_{16} \mathrm{~N}_{2} \mathrm{O}_{2}$ : C, 65.43; H, 7.32; N, 12.72. Found: C, 65.18; H, 7.09; N, 12.62.

2-butyl-2-methyl-malonamide $4 \mathbf{4 j}^{3}{ }^{3}$ white solid, m.p. 176.0-177.0 ${ }^{\circ}$; ${ }^{1} \mathrm{H}$ NMR $(300$ MHz, DMSO): $\delta$ 7.15(s, 2H, $\left.\mathrm{NH}_{2}\right), 7.02\left(\mathrm{~s}, 2 \mathrm{H}, \mathrm{NH}_{2}\right), 1.70-1.64\left(\mathrm{~m}, 2 \mathrm{H}, \mathrm{CH}_{2}\right), 1.26-1.19(\mathrm{~m}$, $\left.5 \mathrm{H}, \mathrm{CH}_{2}, \mathrm{CH}_{3}\right), 1.14-1.06\left(\mathrm{~m}, 2 \mathrm{H}, \mathrm{CH}_{2}\right), 0.83\left(\mathrm{t}, 3 \mathrm{H}, \mathrm{J}=7.2 \mathrm{~Hz}, \mathrm{CH}_{3}\right) ; \mathrm{IR}(\mathrm{KBr}): v 3387$, 3232(NH), 1693(C=O), 1641(C=O); MS m/z (\%): 155( $\left.\mathrm{M}^{+}-17,1\right), 129\left(\mathrm{M}^{+}+1-\mathrm{CONH}_{2}, 29\right)$, $116\left(\mathrm{M}^{+}-\mathrm{Bu}, 33\right), 86(100)$.

2-benzyl-2-ethyl-malonamide 4k. white solid, m.p. $173.4-174.4^{\circ} \mathrm{C}$, Lit. $^{4} 190-194{ }^{\circ} \mathrm{C} ;{ }^{1} \mathrm{H}$ NMR (300 MHz, DMSO): $\delta$ 7.31(br s, 2H, NH $), 7.27-7.12\left(\mathrm{~m}, 7 \mathrm{H}, \mathrm{ArH}, \mathrm{NH}_{2}\right), 3.09(\mathrm{~s}, 2 \mathrm{H}$, $\left.\mathrm{CH}_{2}\right), 1.73\left(\mathrm{q}, 2 \mathrm{H}, \mathrm{J}=7.4 \mathrm{~Hz}, \mathrm{CH}_{2}\right), 0.78\left(\mathrm{t}, 3 \mathrm{H}, \mathrm{J}=7.4 \mathrm{~Hz}, \mathrm{CH}_{3}\right) ; \mathrm{IR}(\mathrm{KBr}): v 3382,3210(\mathrm{NH})$, 1692(C=O); MS m/z (\%): 220(M $\left.\mathrm{M}^{+}, 5\right), 203(5), 177(16), 148(100), 131(50), 91(92.4)$, 65(15.0), 44(14.5).

2-butyl-2-ethyl-malonamide 4l. white solid, m.p. $180.9-181.9^{\circ} \mathrm{C}$, Lit. ${ }^{4} 185^{\circ} \mathrm{C} ;{ }^{1} \mathrm{H}$ NMR (300 MHz, DMSO): $\delta 7.70\left(\mathrm{~s}, 2 \mathrm{H}, \mathrm{NH}_{2}\right), 7.13\left(\mathrm{~s}, 2 \mathrm{H}, \mathrm{NH}_{2}\right), 1.77-1.67\left(\mathrm{~m}, 4 \mathrm{H}, 2 \mathrm{CH}_{2}\right), 1.25-$ 1.18(m, $\left.2 \mathrm{H}, \mathrm{CH}_{2}\right), 1.88-1.01\left(\mathrm{~m}, 2 \mathrm{H}, \mathrm{CH}_{2}\right), 0.82\left(\mathrm{t}, 3 \mathrm{H}, \mathrm{J}=7.2 \mathrm{~Hz}, \mathrm{CH}_{3}\right), 0.68(\mathrm{t}, 3 \mathrm{H}, \mathrm{J}=7.2 \mathrm{~Hz}$, $\left.\mathrm{CH}_{3}\right)$; IR (KBr): v 3387, 3213(NH), 1693(C=O), 1641(C=O); MS m/z (\%): 169(M+17, 2), $143\left(\mathrm{M}^{+}+1-\mathrm{CONH}_{2}, 35\right), 130\left(\mathrm{M}^{+}-\mathrm{Bu}, 22\right), 100(100)$. 
2-allyl-2-benzyl-malonamide $4 \mathbf{m}$. white solid, ${ }^{1} \mathrm{H}$ NMR (300 MHz, DMSO): $\delta 7.60(\mathrm{br}$ $\left.\mathrm{s}, 2 \mathrm{H}, \mathrm{NH}_{2}\right), 7.25-7.15\left(\mathrm{~m}, 7 \mathrm{H}, \mathrm{ArH}, \mathrm{NH}_{2}\right), 5.74-5.60(\mathrm{~m}, 1 \mathrm{H},=\mathrm{CH}), 5.09-5.04(\mathrm{~m}, 2 \mathrm{H}$, $\left.=\mathrm{CH}_{2}\right), 3.10\left(\mathrm{~s}, 2 \mathrm{H}, \mathrm{CH}_{2}\right), 2.49-2.46\left(\mathrm{~m}, 2 \mathrm{H}, \mathrm{CH}_{2}\right)$; IR $(\mathrm{KBr}): v 3379,3223(\mathrm{NH}), 1691$, 1641; MS m/z (\%): 232(M+1), 191(100), 188(28), 174(80), 91(75).

\section{Hydrolysis Products of $\alpha, \alpha$-Disubstituted Malonamides}

(R)-2-(2'-chlorobenzyl)-2-methyl-malonamic acid 5b. white solid, m.p. 104.6-105.6 ${ }^{\circ} \mathrm{C}$; $[\alpha]_{\mathrm{D}}^{25}-16.93$ (c 1.14, MeOH), 97\%ee; ${ }^{1} \mathrm{H}$ NMR (300 MHz, [D $]$ ]acetone): $\delta 12.0$ (br s, $1 \mathrm{H}$, $\mathrm{OH}), 7.42-7.18(\mathrm{~m}, 5 \mathrm{H}, \mathrm{ArH}, \mathrm{NH}), 6.85(\mathrm{br} \mathrm{s}, 1 \mathrm{H}, \mathrm{NH}), 3.47\left(\mathrm{~s}, 2 \mathrm{H}, \mathrm{CH}_{2}\right), 1.41\left(\mathrm{~s}, 3 \mathrm{H}, \mathrm{CH}_{3}\right)$; IR (KBr): v 3475, $3325(\mathrm{NH}), 3000-2850$ (br OH), 1698(C=O); MS m/z (\%): $206\left(\mathrm{M}^{+}-\mathrm{Cl}\right.$, 73), 162(100); Anal. Calcd. for $\mathrm{C}_{11} \mathrm{H}_{12} \mathrm{NO}_{3} \mathrm{Cl}$ : C, 54.67; H, 5.00; N, 5.80; Cl, 14.67. Found: C, 54.71; H, 5.11; N, 5.74; Cl, 14.59.

(R)-2-(3'-chlorobenzyl)-2-methyl-malonamic acid 5c. white solid, m.p. 111.1-112. ${ }^{\circ} \mathrm{C}$; $[\alpha]_{\mathrm{D}}^{25}-9.63(\mathrm{c} 1.25, \mathrm{MeOH}), 95 \% \mathrm{ee} ;{ }^{1} \mathrm{H} \mathrm{NMR}\left(300 \mathrm{MHz},\left[\mathrm{D}_{6}\right]\right.$ acetone): $\delta 12.2(\mathrm{br} \mathrm{s}, 1 \mathrm{H}$, $\mathrm{OH}), 7.32-7.18(\mathrm{~m}, 5 \mathrm{H}, \mathrm{ArH}, \mathrm{NH}), 6.90(\mathrm{br} \mathrm{s}, 1 \mathrm{H}, \mathrm{NH}), 3.24\left(\mathrm{~s}, 2 \mathrm{H}, \mathrm{CH}_{2}\right), 1.42\left(\mathrm{~s}, 3 \mathrm{H}, \mathrm{CH}_{3}\right)$; $\mathrm{IR}(\mathrm{KBr})$ : v 3454, 3331(NH), 3084(br, OH), 1749, 1670(C=O); $\mathrm{MS} \mathrm{m/z}(\%): 243\left(\mathrm{M}^{+}+2,3\right)$, 241( $\left.\mathrm{M}^{+}, 11\right)$, 44(100); Anal. Calcd. for $\mathrm{C}_{11} \mathrm{H}_{12} \mathrm{NO}_{3} \mathrm{Cl}$ : C, 54.67; H, 5.00; N, 5.80; Cl, 14.67. Found: C, 54.95; H, 5.07; N, 5.71; Cl, 14.76.

(R)-2-(4'-chlorobenzyl)-2-methyl-malonamic acid 5d. white solid, m.p. $114.8-115.8^{\circ} \mathrm{C}$; $[\alpha]_{\mathrm{D}}^{21}-12.99(\mathrm{c} 0.83, \mathrm{MeOH}),>99 \% \mathrm{ee} ;{ }^{1} \mathrm{H}$ NMR $\left(300 \mathrm{MHz},\left[\mathrm{D}_{6}\right]\right.$ acetone $): \delta 12.1(\mathrm{br} \mathrm{s}, 1 \mathrm{H}$, $\mathrm{OH}), 7.31-7.23(\mathrm{~m}, 5 \mathrm{H}, \mathrm{ArH}, \mathrm{NH}), 6.91(\mathrm{br} \mathrm{s}, 1 \mathrm{H}, \mathrm{NH}), 3.25(\mathrm{~d}, 1 \mathrm{H}, \mathrm{J}=13.8 \mathrm{~Hz}, \mathrm{CH}), 3.20(\mathrm{~d}$, $1 \mathrm{H}, \mathrm{J}=13.2 \mathrm{~Hz}, \mathrm{CH}), 1.41\left(\mathrm{~s}, 3 \mathrm{H}, \mathrm{CH}_{3}\right) ; \mathrm{IR}(\mathrm{KBr}): v$ 3403, 3227(NH), 2800-3300(br OH), 1727, 1688(C=O); MS m/z (\%): 243( $\left.\mathrm{M}^{+}+2,1\right), 241\left(\mathrm{M}^{+}, 1\right), 197\left(\mathrm{M}^{+}-\mathrm{CONH} 2,25\right), 127(33)$, 125(100); Anal. Calcd. for $\mathrm{C}_{11} \mathrm{H}_{12} \mathrm{NO}_{3} \mathrm{Cl}$ : C, 54.67; H, 5.00; N, 5.80; Cl, 14.67. Found: C, 54.72; H, 5.22; N, 5.61; Cl, 14.86.

(R)-2-(4'-methylbenzyl)-2-methyl-malonamic acid 5e. white solid, m.p. 182.6-183.6 ${ }^{\circ} \mathrm{C}$; $[\alpha]_{\mathrm{D}}{ }^{21}-11.18(\mathrm{c} 0.96, \mathrm{MeOH}),>99 \%$ ee; ${ }^{1} \mathrm{H}$ NMR (300 MHz, [D $]$ acetone): $\delta 12.4(\mathrm{br} \mathrm{s}, 1 \mathrm{H}$, $\mathrm{OH}), 7.27$ (brs, $1 \mathrm{H}, \mathrm{NH}), 7.11,7.05(\mathrm{AB}, 4 \mathrm{H}, \mathrm{J}=8.1 \mathrm{~Hz}, \mathrm{ArH}), 6.91$ (brs, $1 \mathrm{H}, \mathrm{NH}), 3.19$ (s, 2H, $\left.\mathrm{CH}_{2}\right), 2.27\left(\mathrm{~s}, 3 \mathrm{H}, \mathrm{CH}_{3}\right), 1.41\left(\mathrm{~s}, 3 \mathrm{H}, \mathrm{CH}_{3}\right)$; IR( $\left.\mathrm{KBr}\right): v 3451,3329(\mathrm{NH}), 2850-3350(\mathrm{br}, \mathrm{OH})$, 1745, 1670 $(\mathrm{C}=\mathrm{O})$; MS m/z $(\%): 221\left(\mathrm{M}^{+}, 3\right), 105(100)$; Anal. Calcd. for $\mathrm{C}_{12} \mathrm{H}_{15} \mathrm{NO}_{3}$ : C, 65.14; H, 6.83; N, 6.33. Found: C, 65.11; H, 6.92; N, 6.20.

(R)-2-(4'-methoxybenzyl)-2-methyl-malonamic acid 5f. white solid, m.p. 105.5$106.5^{\circ} \mathrm{C} ;[\alpha]_{\mathrm{D}}^{22}-10.75$ (c 1.06, MeOH), >99\%ee; ${ }^{1} \mathrm{H}$ NMR (300 MHz, [D $\left.\mathrm{D}_{6}\right]$ acetone): $\delta$ 12.5(br s, 1H, OH), 7.29(brs, 1H, NH), 7.14, 6.81(AB, 4H, J=8.7Hz, ArH), 6.93(brs, 1H, $\mathrm{NH}), 3.75(\mathrm{~s}, 3 \mathrm{H}, \mathrm{OMe}), 3.17\left(\mathrm{~s}, 2 \mathrm{H}, \mathrm{CH}_{2}\right), 1.41\left(\mathrm{~s}, 3 \mathrm{H}, \mathrm{CH}_{3}\right)$; IR(KBr): $v$ 3428, 3358(NH), 2850-3250(br, OH), 1717, 1652(C=O); MS m/z (\%): 237( $\left.\mathrm{M}^{+}, 2\right), 121(100)$. HRMS Calcd. for $\mathrm{C}_{12} \mathrm{H}_{15} \mathrm{NO}_{4}$ : 237.100109. Found: 237.09736.

(R)-2-(4'-florobenzyl)-2-methyl-malonamic acid 5g. white solid, m.p. 116.9-117.9 ${ }^{\circ} \mathrm{C}$; $[\alpha]_{\mathrm{D}}^{21}-9.46(\mathrm{c} 1.46, \mathrm{MeOH}) ;>99 \% \mathrm{ee} ;{ }^{1} \mathrm{H} \mathrm{NMR}$ (300 MHz, [D $]$ acetone): $\delta$ 7.29-7.24(m, 3H, $\mathrm{ArH}, \mathrm{NH}), 7.05-6.99(\mathrm{~m}, 2 \mathrm{H}, \mathrm{ArH}), 6.90(\mathrm{br} \mathrm{s}, 1 \mathrm{H}, \mathrm{NH}), 3.23\left(\mathrm{~s}, 2 \mathrm{H}, \mathrm{CH}_{2}\right), 1.42\left(\mathrm{~s}, 3 \mathrm{H}, \mathrm{CH}_{3}\right)$; $\mathrm{IR}(\mathrm{KBr}): v$ 3445, 3421(NH), 3202(br, OH), 1656(C=O); MS m/z (\%): 226( $\left.\mathrm{M}^{+}+1,5\right)$, 
225(M $\left(\mathrm{M}^{+}\right.$, 4), 109(100); Anal. Calcd. for $\mathrm{C}_{11} \mathrm{H}_{12} \mathrm{NO}_{3} \mathrm{~F}$ : C, 58.66; H, 5.37; N, 6.22; F, 8.44. Found: C, 58.78; H, 5.52; N, 5.88; F, 8.44.

(R)-2-(4'-bromoenzyl)-2-methyl-malonamic acid 5h. white solid, m.p. 119.5-120.5 ${ }^{\circ}$; $[\alpha]_{\mathrm{D}}^{20}-11.41$ (c 1.17, MeOH), >99\%ee; ${ }^{1} \mathrm{H}$ NMR (300 MHz, [D $]$ ]acetone): $\delta 12.0($ br s, $1 \mathrm{H}$, $\mathrm{OH}), 7.43,7.19(\mathrm{AB}, 4 \mathrm{H}, \mathrm{J}=8.1 \mathrm{~Hz}, \mathrm{ArH}), 7.26(\mathrm{br} \mathrm{s}, 1 \mathrm{H}, \mathrm{NH}), 6.93(\mathrm{br} \mathrm{s}, 1 \mathrm{H}, \mathrm{NH}), 3.21(\mathrm{~s}$, $\left.2 \mathrm{H}, \mathrm{CH}_{2}\right), 1.41\left(\mathrm{~s}, 3 \mathrm{H}, \mathrm{CH}_{3}\right)$; IR(KBr): v 3407, 3227(NH), 2800-3200(br OH), 1725, $1685(\mathrm{C}=\mathrm{O})$; $\mathrm{MS} \mathrm{m} / \mathrm{z}(\%): 287\left(\mathrm{M}^{+}+2,9\right), 285\left(\mathrm{M}^{+}, 10\right)$, 43(100); Anal. Calcd. for $\mathrm{C}_{11} \mathrm{H}_{12} \mathrm{NO}_{3} \mathrm{Br}: \mathrm{C}, 46.18 ; \mathrm{H}, 4.23 ; \mathrm{N}, 4.90 ; \mathrm{Br}, 27.93$. Found: C, 46.09; H, 4.20; N, 4.72; Br, 27.64 .

(R)-2-phenylethyl-2-methyl-malonamic acid 5i. white solid, m.p. $104.5-105.5^{\circ} \mathrm{C} ;[\alpha]_{\mathrm{D}}{ }^{15}$ -4.72(c 0.924, $\mathrm{CHCl}_{3}$ ), >99\%ee; ${ }^{1} \mathrm{H}$ NMR $\left(300 \mathrm{MHz},\left[\mathrm{D}_{6}\right]\right.$ acetone): $\delta 12.5(\mathrm{br} \mathrm{s}, 1 \mathrm{H}, \mathrm{OH})$, 7.39(br s, 1H, NH), 7.30-7.15(m, 5H, ArH), 7.05(br s, 1H, NH), 2.62-2.56(m, 2H, $\mathrm{CH}_{2}$ ), 2.21-2.17(m, 2H, $\left.\mathrm{CH}_{2}\right), 1.53\left(\mathrm{~s}, 3 \mathrm{H}, \mathrm{CH}_{3}\right)$; IR(KBr): v 3449, 3313, 3250(NH), 2500-3250(br $\mathrm{OH}), 1710,1650(\mathrm{C}=\mathrm{O})$; MS $\mathrm{m} / z(\%): 222\left(\mathrm{M}^{+}+1,11\right), 178\left(\mathrm{M}^{+}-\mathrm{CONH}_{2}, 32\right), 73(100)$; Anal. Calcd. for $\mathrm{C}_{12} \mathrm{H}_{15} \mathrm{NO}_{3}$ : C, 65.14; H, 6.83; N, 6.33. Found: C, 65.16; H, 6.83; N, 6.16.

(R)-2-butyl-2-methyl-malonamic acid 5j. white solid, m.p. $84.2-85.2^{\circ} \mathrm{C}$, Lit. $^{3} 90^{\circ} \mathrm{C}$; $[\alpha]_{\mathrm{D}}^{25}-11.05(\mathrm{c} 0.867, \mathrm{MeOH}), 91 \%$ ee $\left\{\right.$ Lit. $\left.^{3}[\alpha]_{\mathrm{D}}{ }^{26}-9.8\left(\mathrm{c} 1.0, \mathrm{CHCl}_{3}\right), 96 \% \mathrm{ee}, \mathrm{R}\right\} ;{ }^{1} \mathrm{H} \mathrm{NMR}$ $\left(300 \mathrm{MHz}, \mathrm{CDCl}_{3}\right.$ ): $\delta$ 8.45(br s, $\left.1 \mathrm{H}, \mathrm{OH}\right), 7.21($ br s, $1 \mathrm{H}, \mathrm{NH}), 7.09($ br s, $1 \mathrm{H}, \mathrm{NH}), 1.95-$ 1.85(m, $\left.2 \mathrm{H}, \mathrm{CH}_{2}\right), 1.49\left(\mathrm{~s}, 3 \mathrm{H}, \mathrm{CH}_{3}\right), 1.40-1.25\left(\mathrm{~m}, 4 \mathrm{H}, 2 \mathrm{CH}_{2}\right), 0.90\left(\mathrm{t}, 3 \mathrm{H}, \mathrm{J}=6.8 \mathrm{~Hz}, \mathrm{CH}_{3}\right)$; $\mathrm{IR}(\mathrm{KBr}): v$ 3410, 3228(NH), 3200-3000(br OH), 1722(C=O), 1677(C=O); MS m/z (\%): $156\left(\mathrm{M}^{+}-17,1.8\right), 130\left(\mathrm{M}^{+}+1-\mathrm{CONH}_{2}, 33.8\right), 129(8.9), 117\left(\mathrm{M}^{+}-\mathrm{Bu}, 56.2\right), 99(28.5), 87(100)$, 86(50.6).

2-allyl-phenylpropionate $6 .{ }^{5}$ Oil, ${ }^{1} \mathrm{H}$ NMR $\left(300 \mathrm{MHz}, \mathrm{CDCl}_{3}\right): \delta 10.0(\mathrm{br} \mathrm{s}, 1 \mathrm{H}, \mathrm{OH})$, 7.32-7.17(m, $5 \mathrm{H}, \mathrm{ArH}), 5.85-5.71(\mathrm{~m}, 1 \mathrm{H}=\mathrm{CH}), 5.13-5.06\left(\mathrm{~m}, 2 \mathrm{H},=\mathrm{CH}_{2}\right), 3.03-2.94(\mathrm{~m}, 1 \mathrm{H}$, $\mathrm{CH}), 2.82-2.72\left(\mathrm{~m}, 2 \mathrm{H}, \mathrm{CH}_{2}\right), 2.44-2.25\left(\mathrm{~m}, 2 \mathrm{H}, \mathrm{CH}_{2}\right)$; IR (film): $v$ 3200-2850(br $\mathrm{OH}$ ), 1708(C=O); MS m/z (\%): 190(M+ 4$), 172(4), 148(19), 147(16), 144(22), 91(100)$.

\section{Hydrolysis of 1 by Rhodococcus sp. CGMCC 0497}

A suspension of $10 \mathrm{~g}$ washed wet cells and $80 \mathrm{ml} 0.1 \mathrm{mM}$ potassium phosphate buffer $(\mathrm{pH}=7.0)$ was incubated at $30^{\circ} \mathrm{C}$ for $30 \mathrm{mins}$ with continuously magnetic stirring before the addition of the substrate, a solution of $100 \mathrm{mg} \mathbf{1}$ dissolved in $100 \mu 1$ acetone. The reaction was quenched by centrifugation. The resulting supernatant was basified with $2 \mathrm{~N} \mathrm{NaOH}$ to $\mathrm{pH}=12$, and extracted with diethyl ether. The organic solutions, after drying $\left(\mathrm{MgSO}_{4}\right)$ and concentration, gave the $\mathbf{2}$ and $\mathbf{4}$. Separation was effected by column chromatography. The aqueous solution was then acidified using $3 \mathrm{~N} \mathrm{HCl}$ to $\mathrm{pH}=2$ and extracted with diethyl ether. Acid 3 and 5 was obtained after removal of the solvent under reduced pressure and purification by flash chromatography on silica gel (elute: petroleum ether/EtOAc/AcOH 150:100:1). The enantiomeric excess of 2 was determined directly by Chiral HPLC. 3 was converted to the corresponding methyl or ethyl esters and then subjected to Chiral HPLC.

(S)-2-cyano-2-methyl-3-phenylpropanamide 2. white solid, m.p. $96.5-97.5^{\circ} \mathrm{C} ;[\alpha]_{\mathrm{D}}{ }^{19}$ +45.2 (c $0.80, \mathrm{CHCl}_{3}$ ), 93\%ee; Enantiomeric excess was determined by HPLC on a Chiralcel OD column with hexane/2-propanol mixtures 8:2; ${ }^{1} \mathrm{H}$ NMR $\left(300 \mathrm{MHz}, \mathrm{CDCl}_{3}\right): \delta$ 
7.40-7.20(m, 5H, Ar-H), 6.04(s, br, 1H, NH), 5.52(s, br, 1H, NH), 3.26(d, 1H, J=13.5Hz, $\mathrm{CH}), 2.96(\mathrm{~d}, 1 \mathrm{H}, \mathrm{J}=13.5 \mathrm{~Hz}, \mathrm{CH}), 1.66\left(\mathrm{~s}, 3 \mathrm{H}, \mathrm{CH}_{3}\right) ; \mathrm{IR}(\mathrm{KBr}): \mathrm{v} 3378,3320(\mathrm{NH})$, 2244(CN), 1666 $(\mathrm{C}=\mathrm{O})$; $\mathrm{MS} m / z(\%): 189\left(\mathrm{M}^{+}+1,3\right), 188\left(\mathrm{M}^{+}, 21\right), 173\left(\mathrm{M}^{+}-\mathrm{CH}_{3}, 4\right)$, 144( $\left(\mathrm{M}^{+}-\mathrm{CONH}_{2}, 19\right), 91(100)$; Anal. Calcd. for $\mathrm{C}_{11} \mathrm{H}_{12} \mathrm{~N}_{2} \mathrm{O}: \mathrm{C}, 70.19 ; \mathrm{H}, 6.43 ; \mathrm{N}, 14.88$. Found: C, 69.98; H, 6.51; N, 14.81 .

(R)-2-cyano-2-methyl-3-phenylpropanoic acid 3. white solid, m.p. 94.6-95.6 ${ }^{\circ} \mathrm{C}$, Lit. $^{6}$ $84^{\circ} \mathrm{C} ;[\alpha]_{\mathrm{D}}{ }^{25}-15.1\left(\mathrm{c} 0.53, \mathrm{CHCl}_{3}\right), 52 \%$ ee, $\left\{\right.$ Lit. $^{3}[\alpha]_{\mathrm{D}} 27.2$ (c 2, $\mathrm{CHCl}_{3}$ ), S $\} ;{ }^{1} \mathrm{H}$ NMR (300 MHz, $\left.\mathrm{CDCl}_{3}\right): \delta$ 9.83(br s, $\left.1 \mathrm{H}, \mathrm{OH}\right), 7.37-7.28(\mathrm{~m}, 5 \mathrm{H}, \mathrm{Ar}-\mathrm{H}), 3.27(\mathrm{~d}, 1 \mathrm{H}, \mathrm{J}=13.5 \mathrm{~Hz}$, $\mathrm{CH}), 3.07(\mathrm{~d}, 1 \mathrm{H}, \mathrm{J}=13.8 \mathrm{~Hz}), 1.65\left(\mathrm{~s}, 3 \mathrm{H}, \mathrm{CH}_{3}\right)$; IR(KBr): v 3074(br OH), 2263(CN), 1747(C=O); $\mathrm{MS} \mathrm{m} / z(\%)$ : 189( $\left.\mathrm{M}^{+}, 2\right), 174\left(\mathrm{M}^{+}-\mathrm{CH}_{3}, 3\right), 144\left(\mathrm{M}^{+}-\mathrm{COOH}, 3\right), 91(100)$.

\footnotetext{
${ }^{1}$ Westfahl, J. C.; Gresham,T. L. J. Am. Chem. Soc. 1955, 77, 936.

${ }^{2}$ Meyer, H. Monatsh. Chem. 1906, 27, 1093.

${ }^{3}$ Yokoyama, M.; Sugai, T.; Ohta, H. Tetrahedron: Asymmetry 1993, 4, 1081.

${ }^{4}$ Asami, R. Kogyo Kagaku Zasshi 1958, 61, 1241; CA 1962, 314.

5 a) Kim, O.H.; Chung, S. Tetrahedron: Asymmetry, 1999, 10, 3746. b) Corey, E. J.; Lee, D.-H. J. Am. Chem. Soc. 1991, 113, 4026.

${ }^{6}$ Cativiela, C.; Diaz-de-Villegas, M. D.; Galvez, J. A. Tetrahedron: Asymmetry, 1994, 5, 261.
} 